\section{ADOLESCENT MEDICINE}

ECHOCARDIOGRAPHIC ASSESSMENT OF LEPT 1 VENTRICULAR (LV) FUNCTION IN HEALTHY ADOLESCENTS FOLLOWING MAXIMAL SUPINE EXERCISE. Victor C. Baum, Robert A. Englander, Lynne L. Levitsky, Pritzker Sch. of Med., Univ.
of Pediatrics, Chicago, nll.

To determine echocardiographic responses to exercise in normal adolescents, 26 healthy adolescents (ages 10.7-17.7 yrs) had M-mode echocardiograms of the $\mathrm{LV}$ and aorta $(\mathrm{Ao})$ prior to and immediately following maximal exercise on a supine bicycle ergometer. The ventricular septum and LV posterior wall were digitized. Fractional ventricular septum and LV posterior wall were digitized. Fractional shortening (FS), Ao systolic time intervals (PEP/ET) and approximate
velocity of circumferential fiber shortening (Vef) were calculated. velocity of circumferential fiber shortening (Vcf) were calculated.
Maximal rate of contraction and relaxation indexed for maximal diameter ( $\mathrm{dD} / \mathrm{dt}$-sys and $\mathrm{dD} / \mathrm{dt}$-dias, diameters/sec) were determined from the digitized trace. The average of 3 resting cycles was compared to the first adequate post-exercise study. All studies were completed within 3 minutes of cessation of exercise. Data are mean \pm SE. All values changed significantly following exercise $(p<.001)$.

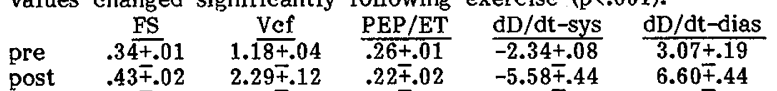

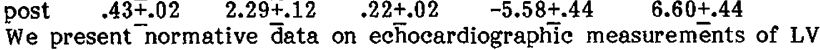
function in healthy adolescents following maximal supine exercise against which data from patients with suspected $L V$ dysfunction can be compared. These data differ from those reported from adults during sub-maximal exercise. This technique is simple, does not involve radiation, and thus may be applied to relatively normal pediatric populations.

EFFECTS OF REFEEDING ON BODY HABITUS IN MAL2 NOURISHED ADOLESCENTS WITH ANOREXIA NERVOSA. Marjorie A. Boeck, George J.Schwartz, AlbertEinstein Coll. Med., Montefiore M.C., Dept. of Peds., Bronx, N.Y.

Weight gain is one of the therapeutic goals for adolescents with Weight gain is one of the therapeutic goals for adolescents with
anorexia nervosa. The quality of such weight gain in terms of tissue deposition has not been studied. We determined the distribution of weight gain between fat and muscle tissue upon refeeding. Percent body fat (from skin fold thickness), \% muscle mass (from creatinine excretion) and muscular development (from corrected upper arm diameter) were obtained on 6 malnourished teenagers initially and upon refeeding. average weight gain was $5.9 \pm 1.3(\mathrm{SE}) \mathrm{kg}$ age from $13-19$ years $(x=15)$; wasted and gained the most weight $(43 \%$ and $23 \%$ of initial weight, respectively) put on a disproportionate amount of body fat $(285,263 \%)$. respectively) put on a disproportionate amount of body fat $(285,263 \%)$. constant and considerably below normal for age/sex. The remaining 4 pts. increased their body weight by $8-12 \%$ and gained both muscle and fot in similar proportions: the \% body fat increased by $27 \pm 6$ (SE)\% and muscle mass by $20 \pm 7 \%$. Muscular development calculated from the square of the increment in upper arm diameter increased by $16 \pm 4 \%$.

We conclude that there are at least two patterns of weight gain with refeeding. Pts. who were extremely malnourished with severe fat and muscle wasting gained a disproportionate amount of fat, while those who were less malnourished gained by the accretion of similar amounts of fat and muscle. Reasons for the observed differences may relate to and muscle. Reasons for the observed differences may relate to of exercise. Therapeutic goals should consider the quality, as well as quantity, of weight gain achieved during refeeding.

CALCIUM AND BONE MINERAL STATUS OF LACTATING TEENS 3 CONSUMING VARIOUS CALCIUM INTAKES. Gary M. Chan, Kris Westover, Martha McMurry. University of Utah, Department of Pediatrics, Salt Lake City, Utah.

Lactating adolescent mothers have been shown to have decreased bone mineral content (BMC). The present study was to evaluate an increased dietary calcium ( $\mathrm{Ca}$ ) intake on the $\mathrm{Ca}$ and $\mathrm{BMC}$ of lactating teens. Three groups of women were studied: 14 adolescent (Low Adol) consuming $900 \mathrm{mg} \mathrm{Ca}$ (56\% RDA), 20 adolescents (High Adol) consuming 1600 or $2000 \mathrm{mg}$ ( 100 or $125 \%$ RDA), and 12 adults consuming $1200 \mathrm{mg}$ (100\% RDA). All lactating subjects were studied at 2,8 and 16 wks postpartum. Serum $\mathrm{Ca}$, phosphorus, magnesium, alkaline phosphatase, 25-OH vitamin D phosphorus, magnesium, alkaline phosphatase, 25-OH vitamin normal for all 3 groups. By $16 \mathrm{wks}$, the CT levels were higher in the Low Adol group compared to the High Adol and adult groups $(13.78 \pm 5.06, M \pm S D$ vs $9.94 \pm 8.12,9.13 \pm 3.87 \mathrm{pg} / \mathrm{ml}, \mathrm{p}<.05)$. PTH and CT levels increased during lactation for the Low Adol while the other 2 groups remained unchanged. BMC was similar at 2 wks, but after 16 wks, the Low Adol group had a decrease in BMC of $10 \%(\mathrm{p}<.05)$. This was significantly different than the High Adol and adult groups which maintained their BMC. All adol dietary $\mathrm{Ca}$ and $\mathrm{P}$ intakes were correlated with changes in their BMC during lactation ( $r=0.45, p<0.01$ for $C a ; r=0.47, p<.01$ for $P)$. By 16 wks, the adult group had a higher BMC than either the Low Adol $(1.003$ vs $0.866, p<.01)$ or the High Adol $(0.899, p<.02)$ groups. These results suggest that bone loss during lactation for teens is accompanied by increased PTH and CT levels and that a high $\mathrm{Ca}$ intake appears to protect against this bone loss.
LANGUAGE DEIAY IN 2 YR, OLD CHILDREN OF

$\uparrow 4$ ADOLESCENT MOTHERS. Cynthia Garcia Coll, Lori Van Houten, Betty $\frac{R}{\text {. Vohr, and William oh. Brown }}$, Univ., Women \& Infants Hosp., Dept. of Ped., Providence; RI. We have previously reported that adolescent mothers are more physical and less verbal with their infants, especially while teaching difficult tasks, and that their infants vocalize less by 8 months of age. Are children of adolescent mothers at risk for language delay? The present study hypothesized that $2 \mathrm{yr}$. old children of adolescent mothers (CAM) would have lower receptive and expressive language scores and that these scores would be related to multiple social and demographic high risk factors. Twenty primiparous, Caucasian, low to middle class mothers (half $\leq 17$ years of age at child's birth) and their 2 yr. old children were studied. The Caldwell Home Inventory was scored by an unbiased observer during a two-hour home visit. The Bayley Scales (MDI and PDI) and the Mullen Scales of Early Learning (Language Expressive (LEO) and Language Receptive (LRO)) were administered by another unbiased examiner during a clinic visit. Tympanography was performed and sociodemographic information was obtained. CAM had lower LRO $(p<.01)$ and LEO $(\mathrm{p}<.001)$ scores, but no differences were observed in MDI, PDI and tympanograms. Children whose mothers possess 2 or more of the following: + maternal age, + SES, teducation, + family support, or $\downarrow$ HOME total score had lower LRO $(p<.001)$ and LEO $(p<.01)$ scores. We conclude that $\downarrow$ CAM are at risk for language delay which is related to multiple social and demograph1c risk factors associated with adolescent childbearing.

BEACH PARTY: DEPRESSION IN ADOLESCENTS AND YOUNG 5 ADULTS IN A RESORT COMMUNITY. Carolyn L. GouTd Felix P. Heald). From the Department of Pediatrics, University of Maryiand, School of Medicine, Baltimore.

Depression and suicide are recognized as major problems among adolescents and young adults. Little is known of the epidemiology and risk factors associated with depression. We studied the prevalence of depression in a presumably low risk group. The study was conducted at a general youth clinic in ocean $\dot{C} i t y$, Maryland between June 3,1984 and September 2, 1984. This clinic serves teen and young adult summer workers, vacationers and some permanent residents. All patients between the ages of 13 and 25 were asked to complete the CES-D depression scale. This is a standardized survey instrument which measures depressive symptomatology among general poputations. Demographic data and diagnosis were abstracted from the medical record. 587 patients returned a valid questionnaire. Data collection was complete for $94 \%$ of the questionnaires. Overa $11,29 \%$ of our sample scored in the depressed range. This is significantly greater than the $17 \%$ noted in the general population $(p<0.05)$. Depression was not related to the duration of residence at the resort. It was also unrelated to the patient's socioeconomic resort. Itus, educational level or age. Females were significantly more depressed than males. The only diagnosis which was signifmore depressed than males. The only diagnosis which was signif-
icantly related to depression was pregnancy. We conclude that depressive symptomatology is a significant problem among adolescents and young aduits but that its presence in specific patients is not predictable.

CONTRACEPTIVE COMPLIANCE IN THE PRIVATE SECTOR,

6 'Estherann Grace, S. Jean Emans, Elizabeth R. Wood Harvard Medical School, Boston, MA.

Erratic contraceptive usage is a major factor in teenage pregnancy. Many studies have addressed the predictors of pregnancy. Many studles have addressed the predictors of non-compliance in the use of oral contraceptives in adolescent clinics. This study was designed to focus on the previousiy ignored private sector, examining their contraceptive behaviors and predictors of their compliance. Patients from a suburban private practice of adolescent medicine were compared to the
adolescent clinic population of The Children's Hospital. Profiles commonly associated with contraceptive compliance were recorded at the initial and return visits. Study subjects were randomly assigned to 2 groups receiving either simplified or detailed instructions on pill usage and management of bleeding and amenorrhea. All patients were provided 3 packs of voriny $1+35$ and scheduled for a 3 month return visit. 51 private and 60 clinic patients (15-22 yrs) were studied. Of the private and 60 clinic patients (15-22 yrs) were studied. Of the private patients 42 made their own appointments, 29 without visit, $30 \%$ having never used contraception. $82 \%$ of private and $47 \%$ of clinic patients returned at 3 months. Prior completion of a course of antiblotics did not predict 0.C. compliance. The type of instruction (simplified vs. detailed) did not affect pill usage $(p=.10)$. The many epidemlologic factors associated pill usage $(p=.10)$. The many epidemiologic factors associated
with recelving health care in the private sector were major determinants of compliance ( $\mathrm{p}(.001)$. 